\title{
ARTICLE \\ Knockdown of the histone di-methyltransferase G9a in nucleus accumbens shell decreases cocaine self-administration, stress-induced reinstatement, and anxiety
}

 \\ David W. Self ${ }^{1}$
}

\begin{abstract}
Comorbid neuropsychiatric disorders such as addiction and anxiety could involve common underlying mechanisms. One potential mechanism involves epigenetic regulation of histone 3 dimethylation at lysine 9 residues (H3K9me2) by the histone dimethyltransferase G9a. Here we provide evidence that local AAV-RNAi-mediated knockdown of G9a expression in nucleus accumbens shell (NACSh) of male rats reduces both addictive-related and anxiety-related behaviors. Specifically, G9a knockdown reduces sensitivity to low dose cocaine reinforcement when cocaine is freely available (fixed ratio schedule). Similarly, G9a knockdown reduces motivation for cocaine under higher effort demands (progressive ratio schedule). Following several weeks of forced abstinence, G9a knockdown attenuates extinction responding and reinstatement triggered by either cocaine-priming injections or footshock stress. This decrease in addictive behavior is associated with a long-term reduction in anxiety-like behavior as measured by the elevated plus maze (EPM). G9a knockdown also reduces basal anxiety-like behavior in EPM and marble burying tests in drug-naïve rats. These results complement our previous work showing that increased G9a expression in NAcSh enhances addictive-related and anxiety-related behaviors, indicating that G9a bi-directionally controls these responses. These results also suggest that regulation of G9a-influenced gene expression could be a common epigenetic mechanism for co-morbid anxiety and psychostimulant addiction.
\end{abstract}

Neuropsychopharmacology (2019) 44:1370-1376; https://doi.org/10.1038/s41386-018-0305-4

\section{INTRODUCTION}

The ability to successfully treat addiction is complicated by the existence of co-morbid neuropsychiatric conditions such as anxiety [1, 2]. The underlying causes for co-morbid addiction and anxiety are still unknown, and there is a great need for better clinical treatments for both disorders [3-5]. One potential mechanism that could underlie the development of comorbid addiction and anxiety may be epigenetic changes that alter experience-dependent regulation of gene expression. These epigenetic changes can in turn account for lasting changes in behavioral states or responses to drugs and stress. For instance, past drug use alters the behavioral response to chronic social defeat stress [6] or stress-induced drug reinstatement [7] through alterations in epigenetic regulation of histone methylation and acetylation, DNA methylation, or related mechanisms [8].

Drug addiction in particular may involve regulation of the histone di-methyltransferase G9a (also called EHMT2 and KMT1C) [9]. G9a and/or H3K9me2 levels are reduced in the nucleus accumbens (NAc) following exposure to numerous drugs of abuse, including cocaine $[10,11]$ and morphine $[12]$. It is also altered by alcohol in primary neuronal cultures [13], suggesting a common epigenetic change associated with drug addiction. G9a-mediated dimethylation of H3K9 generally acts to suppress numerous genes relevant to addiction, including BDNF [14]—thus, it may play a role in cocaine self-administration changes produced by manipulating BDNF-TrkB signaling in this brain region [15-17]. Initial reports of a direct role for G9a in drug reward are supported by the finding that local G9a knockout in NAc neurons enhances cocaine-conditioned place preference, suggesting that cocaineinduced reductions in G9a levels may contribute to sensitization processes in cocaine addiction. Conversely, G9a overexpression in NAc neurons reduces cocaine-conditioned place preference [11]. However, we recently reported that G9a overexpression in NAC shell (NAcSh) neurons increases motivation for cocaine in selfadministering rats [7]. G9a overexpression also enhances stressinduced reinstatement of cocaine seeking during forced abstinence, suggesting that decreases in NAc G9a levels following repeated cocaine exposure act counter to the addiction process with respect to self-administration behavior [7].

In addition to regulating responses to drugs of abuse, global deletion of forebrain G9a produces a variety of behavioral impairments including decreased learning, exploratory behavior, and sucrose preference. These mice also show decreased anxietylike responses in the elevated plus maze (EPM) [18], although these effects could be attributed to developmental alterations. Similarly, a recent study found that systemic injection of G9a

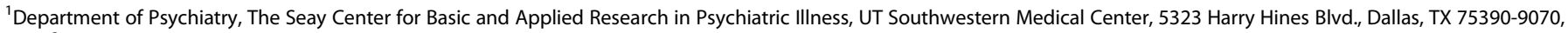

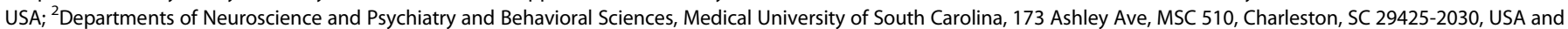
${ }^{3}$ Department of Neuroscience and Friedman Brain Institute, Icahn School of Medicine at Mount Sinai, One Gustave L. Levy Place, Box 1065, New York, NY 10029, USA Correspondence: Ethan M. Anderson (ethan.anderson.phd@gmail.com) 
inhibitors reduces a variety of anxiety-related behaviors like elevated zero maze, open field, and marble burying [19], reflecting the diverse role of G9a-regulated gene expression on these behaviors across multiple brain regions. Conversely, we found that localized, but transient, G9a overexpression in NAcSh leads to a long-lasting increase in anxiety-like behavior [7]. Based on these findings, we hypothesized that mimicking cocaine-induced reductions in G9a levels in the NAcSh would oppose anxietyrelated behavioral responses.

In this study, we find that reducing G9a levels via viralmediated RNA-interference (AAV-RNAi) in NAcSh neurons reduces the motivation for cocaine in self-administering rats and attenuates relapse to cocaine-seeking behavior elicited by both conditioned stimuli and stress. The latter effect is consistent with observable decreases in basal anxiety-like behavior, and complementary to results previously found with G9a overexpression in this brain region [7]. Together, these results suggest that G9a-regulated gene expression in NAcSh neurons exerts strong, bi-directional control over cocaine addiction and anxiety behaviors.

\section{MATERIALS AND METHODS}

Animal use and care

Adult male Sprague-Dawley rats (Charles-River, Kingston, RI, USA) initially weighing $250-300 \mathrm{~g}$ were singly housed in a climatecontrolled environment $\left(21^{\circ} \mathrm{C}\right)$ on a $12 \mathrm{~h}$ light-dark cycle (lights on at 6:00a.m.). Animals were habituated to the housing environment for at least 7 days prior to use in experiments, and had food and water ad libitum, except when operant training with sucrose pellets. All experiments were performed during the light cycle, and were approved by the UTSW or MUSC Institutional Animal Care and Use Committee (IACUC) in facilities accredited by the American Association for the Accreditation of Laboratory Animal Care (AAALAC). All procedures were conducted in accordance with the guidelines established by the National Institutes of Health and the National Research Council.

\section{Vector construction}

An shRNA construct against rat G9a/Ehmt2 (Origene TG713894) was first screened in vitro for knockdown capacity before being selected for further vector construction. This G9a shRNA, as well a negative scrambled (SC) control sequence that does not recognize any sequence in the vertebrate genome, were converted to premiRNA oligos (gagccacctccaggtggttgt and aaatgtactgcgcgtggagac, respectively) and cloned into a BLOCK-iT Pol II shRNAi pcDNA 6.2-GW/EmGFP-miR expression vector (Invitrogen), as per manufacturer instructions. The double stranded miRNA sequences were then subcloned into an AAV-IRES-GFP vector, where the miR and GFP sequences are expressed under a CMV promoter. Plasmids were then packaged into AAV particles (serotype 2) by the UNC viral vector core.

\section{In vitro characterization}

Scrambled and shRNA plasmids were transfected into $70 \%$ confluent PC12 cells using calcium phosphate with 1 ug of DNA plasmid per sample, then either harvested in $100 \mu \mathrm{l}$ Laemmli sample buffer after 3 days or used for imaging at $\times 10$ magnification. A mock transfection with no plasmid was also performed as a control. Cell densities and DAPI-stained nuclei were examined to confirm cell viability.

\section{Rat stereotaxic surgery}

For in vivo AAV characterization and behavioral experiments, rats were anesthetized (ketamine/xylazine, $100 / 10 \mathrm{mg} / \mathrm{kg}$, i.p.) and $1 \mu \mathrm{l}$ vector was infused over $10 \mathrm{~min}$ bilaterally into the NAcSh $\left(10^{\circ}\right.$ angle, $\mathrm{AP}+1.7 \mathrm{~mm}, \mathrm{ML}+/-2.15 \mathrm{~mm}, \mathrm{DV}-6.3 \mathrm{~mm}$, ventral to dura). For the in vivo AAV characterization in Fig. 1d, e, one side of the brain was infused with AAV-SC, the other with AAV-G9a-RNAi in a paired design. Brain tissue was harvested after 3 weeks of AAV

\section{A}
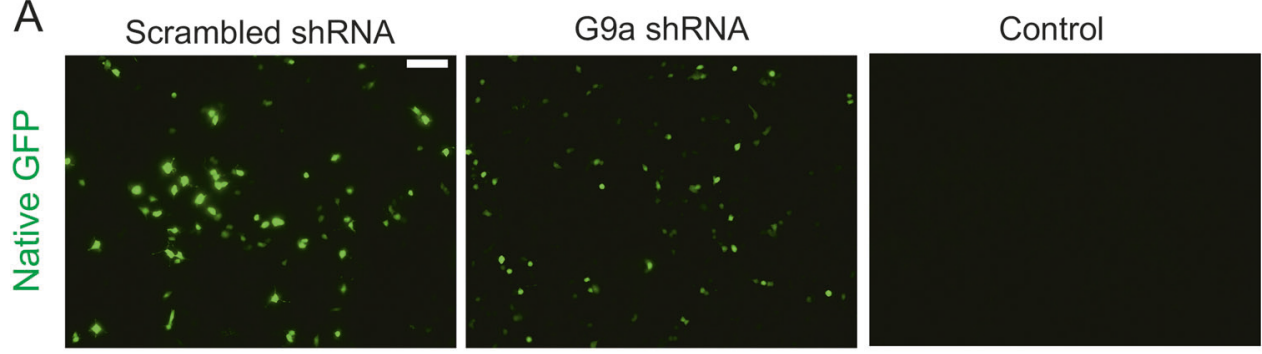

B

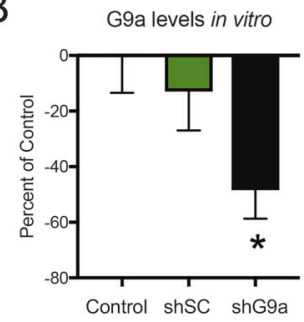

G9a $160 \mathrm{kDa}$

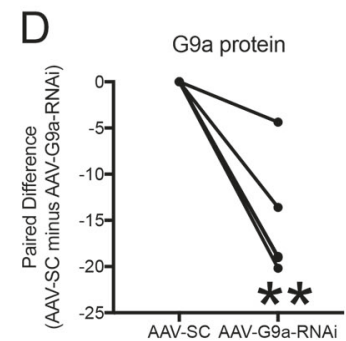

G9a, $160 \mathrm{kDa}$ -

B-tub, $50 \mathrm{kDa}$ -
AAV-G9a-RNAi

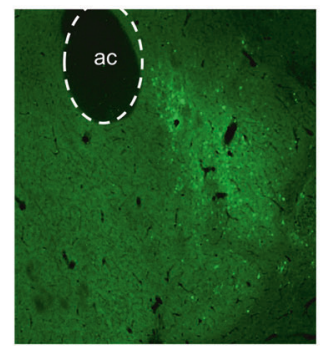

AAV-SC



Fig. 1 G9a knockdown via AAV-G9a-RNAi both in vitro and in NAcSh in vivo. a $\times 10$ images of native GFP expression in PC12 cells following transfection with G9a-scrambled (SC) RNA, the G9a-shRNAi plasmid, or no plasmid as a control (white scale bar equals 100 micron). b Protein levels of G9a in transfected PC12 cells ( $n=5$ for each group). $c \times 4$ Images of NAcSh tissue infected with AAV-G9a-RNAi or AAV-SC in vivo with native GFP fluorescence (white scale bar equals 500 micron, the anterior commissure is circled). d Protein levels of G9a and (e) H3K9 dimethylation (H3K9me2/total H3) in NAcSh after 3 weeks of AAV expression (AAV-SC: $n=5$, AAV-G9a-RNAi: $n=5$ ). Data are expressed as mean $+/-$ s.e.m. ${ }^{*} p<0.05$ and ${ }^{* *} p<0.01$ compared with AAV-SC controls 
expression by taking half-moon punches of NAcSh from chilled $1 \mathrm{~mm}$ thick coronal brain slices ( $+1.7 \mathrm{~mm}$ anterior to bregma) as described [20]. Samples were immediately frozen on dry ice before homogenizing for western blots as described below.

\section{Western blotting}

Blots were run according to the methods in Edwards et al. [21]. except where noted. Primary antibodies: anti-G9a/EHMT2 (RRID: AB_731483, cat: ab40542, abcam, rabbit, 1:4000), anti-Histone H3 (RRID: AB_331563, cat: 9715 S, cell signaling, rabbit, 1:10,000), antiH3K9me2 (RRID: AB_449854, cat: ab1220, abcam, mouse, 1:10,000), and anti- $\beta$-tubulin III (RRID: AB_2313564, cat: TUJ, chicken, 1:50,000). Secondaries: anti-rabbit (RRID: AB_11125142, BioRad, cat: $170-6515,1: 10,000)$ or anti-mouse HRP (RRID: AB_11125547, BioRad, cat: 170-6515, 1:10,000). Blots were first probed for anti-H3K9me2 stripped with Restore buffer (Thermo), re-blocked and re-probed with anti-H3 total. Protein levels were analyzed with ImageJ software.

\section{Cocaine self-administration}

A timeline for this experiment is shown in Fig. 2a. Food training and surgeries were performed as described previously [22]. Cocaine SA (fixed ratio (FR) acquisition/stabilization, withinsession FR dose-responding, and progressive ratio) was performed as described [7]. At the end of both FR and PR dose-response testing phases, catheter patency was confirmed by brief induction of anesthesia with intravenous methohexital injection.

\section{Cocaine abstinence and reinstatement testing}

Following 2 weeks of abstinence (AB) after the PR testing, rats were tested in extinction (EXT) and reinstatement according to previously published methods [23]. Briefly, rats were placed into the same operant chambers, but under extinction conditions (i.e., the levers and house light were present, but lever pressing did not result in cue presentation, house light deactivation, pump activation (i.e., sound cues), or cocaine injections). After 5 days of $3 \mathrm{~h}$ extinction sessions, we then tested reinstatement over 5 consecutive days. First, cue-induced reinstatement was tested over a $1 \mathrm{~h}$ period following $2 \mathrm{~h}$ of additional extinction conditions; drug-paired cue lights and pump activation sounds were presented non-contingently every $2 \mathrm{~min}$ for the first $10 \mathrm{~min}$ of the $1 \mathrm{~h}$ test. Next, reinstatement induced by cocaine priming injections was tested with cocaine $(0,5$, or $15 \mathrm{mg} / \mathrm{kg}$ cocaine, ip) over 3 days in counterbalanced order. Finally, reinstatement induced by intermittent footshock stress was measured for a $1 \mathrm{~h}$ period following $30 \mathrm{~min}$ of random interval shocks $(0.8 \mathrm{mV} / 0.5 \mathrm{~s})$ at an average interval of $30 \mathrm{~s}$.

Elevated plus maze (EPM)

Rats were placed in the center of a black plexiglass EPM as previously described [7] and allowed to explore for $5 \mathrm{~min}$. The animals were monitored from above by a video camera connected to a computer running video tracking software (Ethovision 3.0, Noldus, Leesburg, Virginia) to determine time spent in the open arms.

Basal anxiety measures

The effects of G9a knockdown on basal anxiety-like behavior were tested in drug-naïve rats (see Fig. 5 a for timeline) 3 weeks after AAV infusions to allow peak expression of AAV [24]. EPM was tested as described above, followed by a marble burying test where rats were placed in a cage with extra bedding and 25 marbles arranged in a $5 \times 5$ grid as shown in Fig. $5 \mathrm{~d}$. After $15 \mathrm{~min}$, the number of marbles buried at least $50 \%$ were counted. Finally, locomotor activity was tested for 30 min using VersaMax locomotor boxes.

\section{Placement verification}

Following testing, rats that self-administered cocaine were perfused with $4 \%$ paraformaldehyde for placement verification via GFP immunoreactivity/or native GFP fluorescence. Animals were excluded if GFP spread beyond the NAcSh according to a brain atlas [25], similar to images shown in Fig. 1c. For cocaine SA rats, 1 AAV-SC and 4 AAV-G9a-RNAi rats were removed due to missed placements. For naïve rats, 2 AAV-SC and 3 AAV-G9a-RNAi rats were removed.

Experimental design and statistical analysis

One-way analysis of variance (ANOVA) was used to compare groups for in vitro western blot characterization of G9a levels.

A

Stage

Time

Viral Expression

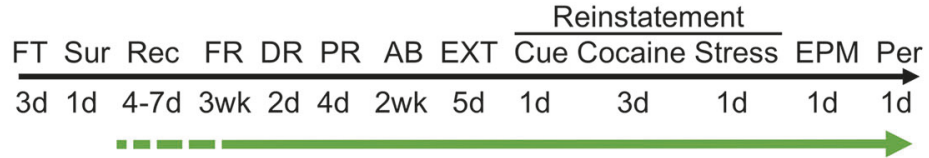

B

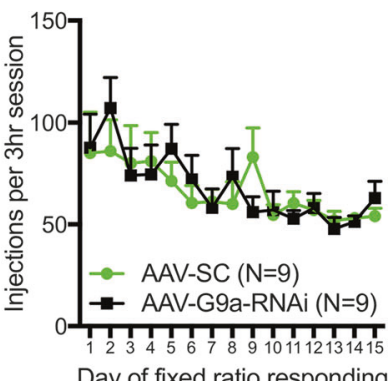

C Dose response (Days 1-2)

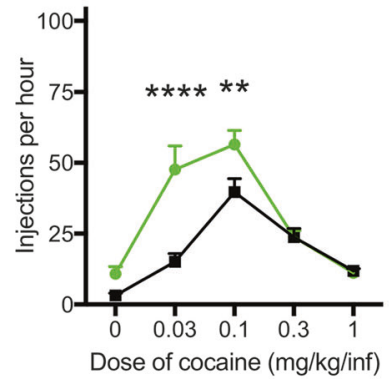

Progressive ratio (Day 2)

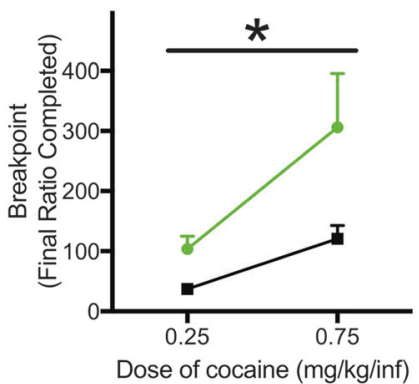

Fig. 2 AAV-RNAi-mediated G9a knockdown in NAcSh decreases cocaine self-administration behavior. a Experimental time course depicting lever-press training with food pellets (FT), surgery (Sur), recovery (Rec), and fixed ratio (FR) cocaine self-administration training, followed by both FR and progressive ratio (PR) dose-response (DR) testing. Extinction (EXT) and reinstatement tests were performed after a 2-week abstinence period (AB). Finally, elevated plus maze (EPM) testing was performed before animals were perfused (Per). The green arrow represents the AAV expression period. b Average cocaine injections in study groups during 3 weeks ( 5 day/week) of acquisition/stabilization of cocaine self-administration $(0.5 \mathrm{mg} / \mathrm{kg} /$ injection). c FR dose-response self-administration and (d) PR dose-response self-administration. AAVSC: $n=9$, AAV-G9a-RNAi: $n=9$. Data are expressed as mean $+/-$ s.e.m. ${ }^{* *} p<0.01$ and ${ }^{* * * *} p<0.0001$ compared with AAV-SC controls. For 2 days, the asterisk represents a significant effect of vector after a repeated measures Two-Way ANOVA 
Paired t-tests were used to compare G9a and H3K9me2 levels in NAcSh after AAV-SC or AAV-G9a-RNAi infusions in vivo. Behavioral data were analyzed with mixed model repeated measures twoway ANOVA. Within-subject factors were time or dose, and between-subject factors were group (AAV-SC vs. AAV-G9a-RNAi). Following significant interactions, vector groups were compared with least significant difference post-hoc tests. All reinstatement tests were measured as the difference in number of drug-paired lever presses during the hour of reinstatement testing minus lever presses during the last hour of extinction prior to testing. AAV-SC vs. AAV-G9a-RNAi in EPM and marble burying tests were compared by $t$-tests. Linear regression was used to compare within-subject behavioral results following a Grubbs' test for outliers. All statistics were performed with Graphpad Prism and a $p<0.05$ was considered significant.

\section{RESULTS}

G9a-RNAi characterization

RNAi-mediated G9a knockdown was first evaluated in transfected PC12 cells in vitro. Fig. 1a shows that both the G9a-RNAi plasmid and the scrambled RNAi control plasmid transfect the cells and express GFP. Cell densities and DAPI-stained nuclei were similar between RNAi and scrambled controls indicating no cell viability issues (data not shown). Western blots of lysed PC12 cells demonstrate that transfected G9a-RNAi significantly decreases G9a protein levels compared to scrambled RNA controls (Fig. 1b, $\left.F_{2,12}=3.903, p=0.0495\right)$. When infused into NAcSh in vivo, both AAV vectors express GFP protein (Fig. 1c), with transduction observed only in cells with neuronal morphology. Western blots of NAcSh tissue found that AAV-G9a-RNAi infusions significantly decrease G9a protein levels after 3 weeks compared to withinsubject (contralateral) AAV-SC control infusions (Fig. 1d, $t_{4}=5.169$, $p=0.0067$ ) while $\beta$-tubulin levels remain unchanged (SC: $100 \pm$ 16.42; AAV-G9a-RNAi: $99.26 \pm 17.76 ; t_{5}=0.1072, p=0.9198$ ). AAVG9a-RNAi also produced a concomitant decrease in H3K9me2 levels (Fig. 1e, $t_{5}=2.901, p=0.0441$ ). Together, these findings confirm that AAV-G9a-RNAi reduces G9a and H3K9 dimethylation levels in NAcSh.

NAcSh G9a knockdown on cocaine self-administration

We tested the hypothesis that G9a knockdown in NAcSh reduces cocaine self-administration and relapse behaviors since we previously found that G9a overexpression increases these behaviors [7]. A timeline for serial testing of these procedures is shown in Fig. 2a. Animals were trained to self-administer cocaine for 15 days and no differences were observed across both AAV study groups (Fig. 2b; Vector: $F_{1,16}=0.03251, p=0.8592$; Time: $F_{14,224}=3.832, p<0.0001$; Interaction: $F_{14,224}=0.7287, \quad p=$ $0.7444)$. However, animals with AAV-RNAi-mediated G9a knockdown exhibit a downward shift in cocaine self-administration at low-moderate cocaine injection doses compared to controls (Fig. 2c; Vector: $F_{1,16}=9.426, p=0.0073$; Dose: $F_{4,64}=55.13, p<$ 0.0001 ; Interaction: $F_{4,64}=9.404, p<0.0001$ ). This indicates that reduced levels of G9a in NAcSh decrease the sensitivity to cocaine reinforcement in self-administering animals. Next, the level of effort rats exert to maintain cocaine self-administration behavior was assessed on the PR reinforcement schedule at two cocaine injection doses that are above the threshold for maintaining selfadministration in FR dose response testing. Fig. 2d shows that AAV-RNAi-mediated knockdown of G9a expression in NAcSh decreases the motivation for cocaine, as indicated by a significant main effect of AAV group for differences in PR breakpoints, i.e., the highest ratio of lever-presses/cocaine injection achieved before animals voluntarily cease self-administration behavior (Vector: $F_{1,16}=5.437, p=0.0331$; Dose: $F_{1,16}=12.77, p=0.0025$; Interaction: $\left.F_{1,16}=2.221, p=0.1556\right)$. These results indicate that $\mathrm{G} 9 \mathrm{a}$ knockdown in the NAcSh decreases the motivation for cocaine, with no effect on cocaine self-administration under relatively free access conditions in this range of injection doses (Fig. 2b).

NAcSh G9a knockdown on drug-seeking behaviors

We next tested the role of G9a on cocaine-seeking behavior after an abstinence period from cocaine self-administration in AAV-G9a-RNAi infected animals and AAV-SC controls (see Fig. 2a for experimental timeline). Animals with G9a knockdown in NAcSh exhibit strong reductions in cocaine-seeking behavior at the drug-paired lever upon initial exposure to the cocaine-paired environmental context in extinction testing (Fig. 3a, Vector: $F_{1,16}=16.78, p=0.0008$; Day: $F_{4,64}=13.65, p<0.0001$; Interaction: $\left.F_{4,64}=5.211, p=0.0011\right)$. In contrast, there are no significant differences between G9a RNAi and control groups for reinstatement elicited by cocaine injection cues, but response rates are relatively low compared to extinction baselines (Fig. 3b, Vector: $F_{1,16}=0.8016, p=0.3839$; Reinstatement: $F_{1,16}=2.590, p=0.1271$; Interaction: $F_{1,16}=0.3966, p=0.5378$ ). However, G9a knockdown in the NAcSh effectively reduces cocaine-primed reinstatement of cocaine seeking across 3 dose conditions (Fig. 3c, Vector: $F_{1,16}=4.698, p=0.0456$; Dose: $F_{2,32}=$ 35.9, $p<0.0001$; Interaction: $F_{2,32}=2.339, p=0.1127$ ). AAV-G9aRNAi-infected rats also display reductions in cocaine seeking following exposure to intermittent footshocks compared to the AAV-SC group (Fig. 3d, Vector: $F_{1,16}=5.874, p=0.0276$; Reinstatement: $F_{1,16}=51.55, p<0.0001$; Interaction: $F_{1,16}=5.874, p=0.0276$ ). Together, these results indicate that reduced G9a in NAcSh neurons attenuates context-related cocaine seeking during the initial extinction testing and reinstatement of drug seeking following cocaine priming or an acute stressful experience.
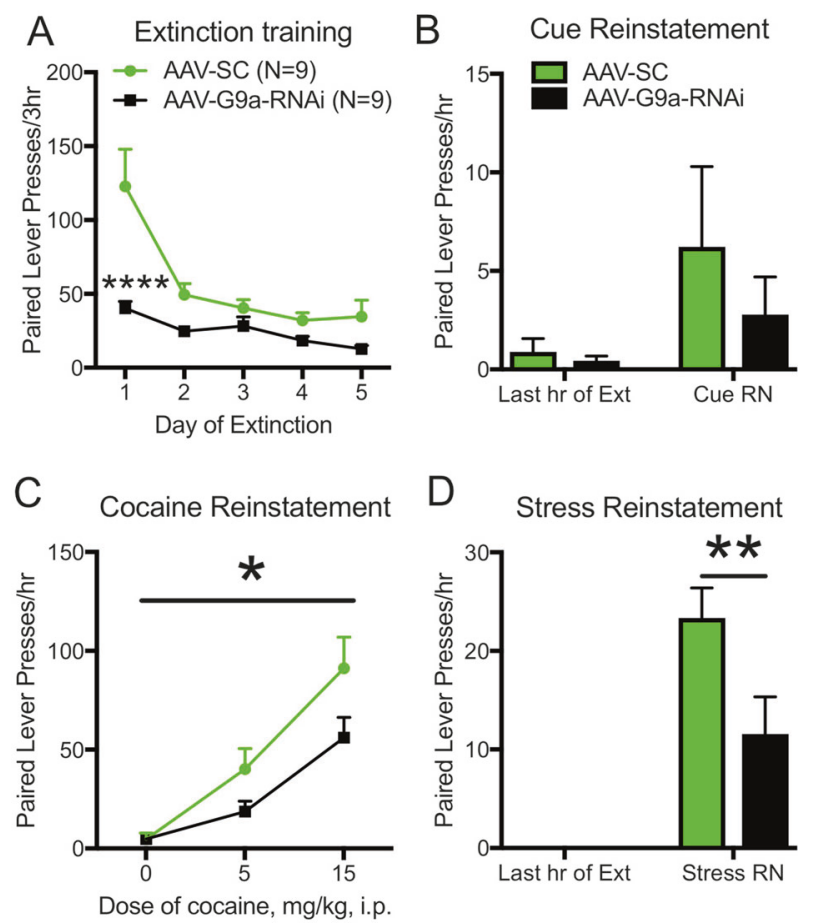

Fig. 3 AAV-RNAi-mediated G9a knockdown in NAcSh reduces cocaine-seeking behavior during extinction, cocaine-primed reinstatement, and intermittent footshock stress-induced reinstatement tests. a Drug-paired lever presses over 5 days of extinction in $3 \mathrm{~h}$ test sessions, (b) during non-contingent light and tone cue presentation, (c) following i.p. injections of saline or cocaine, or (d) after $30 \mathrm{~min}$ of intermittent footshocks (AAV-SC: $n=9$, AAV-G9a-RNAi: $n=9$ ). Data are expressed as mean $+/-$ s.e.m. ${ }^{*} p<0.05,{ }^{* *} p<0.01$, and ${ }^{* * *} p<$ 0.0001 compared with AAV-SC controls. For $3 c$, the asterisk represents a significant effect of vector after a repeated measures two-way ANOVA 
NAcSh G9a knockdown reduces anxiety-like behavior following cocaine self-administration

Given our previous work showing that G9a overexpression in the NAcSh increases anxiety-like behavior based on avoidance of open arms of the EPM [7], we tested the hypothesis that reductions in NAcSh G9a levels would decrease anxiety-like behavior in this test following cocaine self-administration and reinstatement testing. Indeed, animals infected with AAV-G9aRNAi spend more time in the open arms of the EPM than AAV-SC controls (Fig. $4 a, t_{16}=2.332, p=0.0331$ ). No differences in overall locomotor activity were observed in the maze (distance traveled: AAV-SC, $1422 \pm 91.49 \mathrm{~cm}$; AAV-G9a-RNAi, $1432 \pm 118.2 \mathrm{~cm} ; t_{16}=$ $0.07137, p=0.9440$ ). Interestingly, anxiety-like behavior (time spent in the open arm) significantly correlates with cocaine selfadministration breakpoints (high dose) in the progressive ratio task (Fig. $4 \mathrm{~b}, R^{2}=0.4852, F_{1,14}=13.19, p=0.0027$ ). No other behaviors correlate with this anxiety measure, although statistical trends $(p<0.1)$ are noted for extinction responding on test day 1 and the low dose $(0.03 \mathrm{mg} / \mathrm{kg} /$ injection) of fixed ratio cocaine selfadministration (data not shown). These data suggest that the reduction in the motivation for cocaine produced by G9a knockdown might be related to a reduction in basal anxiety.

NAcSh G9a knockdown reduces basal anxiety-like state We next tested whether drug-naïve animals infected with AAV-G9aRNAi would show reduced anxiety-like behavior in EPM and the marble burying test (see Fig. 5a for timeline). Rats with G9a knockdown in the NAcSh spend more time in the open arms of the plus maze (Fig. $5 b, t_{8}=2.561, p=0.0168$ ) and bury fewer marbles than controls (Fig. $5 c$, e-g, $t_{8}=1.988, p=0.0410$ ), but have similar locomotor behavior (distance traveled, AAV-SC, $1744 \pm 187.1 \mathrm{~cm}$; AAV-G9a-RNAi, $1550 \pm 231.2 \mathrm{~cm} ; t_{8}=0.6529, p=0.2661$ ). Of note, the two measures of anxiety-like behavior (open arm time vs. marbles buried) were significantly correlated (Fig. $5 \mathrm{~d}, R^{2}=0.4535$, $F_{1,8}=6.639, p=0.0328$ ). These results confirm that G9a knockdown in the NAcSh induces a pre-existing anxiolytic-like state that might influence the noted decreases in cocaine self-administration and cocaine-seeking behavior (Figs. 2 and 3).

\section{DISCUSSION}

This study found that reducing G9a levels reduces H3K9 dimethylation in NACSh neurons and also decreases many clinically-relevant aspects of cocaine-taking, cocaine-seeking and anxiety-like behaviors. We found that G9a knockdown in the NAcSh decreases sensitivity to low-dose cocaine reinforcement, the motivation to work for cocaine, and the ability of contextual (extinction), pharmacological, and stressful stimuli to induce cocaine seeking after a period of forced abstinence. These findings are bolstered by the findings that opposite effects are produced by overexpressing G9a in NAcSh via a transientlyexpressing HSV vector [7]. In this previous study, we found that increasing G9a levels in NAcSh increased H3K9 dimethylation and enhanced sensitivity to, and motivation for, cocaine in selfadministering rats. Transient G9a overexpression limited to the cocaine self-administration phase also led to an enduring enhancement in intermittent footshock stress (but not cueprimed or cocaine-primed) reinstatement of cocaine-seeking behavior, even long-after G9a and H3K9me2 levels return to normal. Taken together, G9a clearly exerts bi-directionally control of numerous aspects of cocaine-related behaviors, and these behaviors track closely with changes in basal anxiety state.

Although the behavioral effects of RNAi-mediated knockdown of G9a in the NAcSh are generally opposite to those produced by transient G9a overexpression [7], there are several notable differences. For example, transient HSV-mediated G9a overexpression produces no enduring changes in extinction responding or cocaine-induced cocaine-seeking behavior, and the long-term
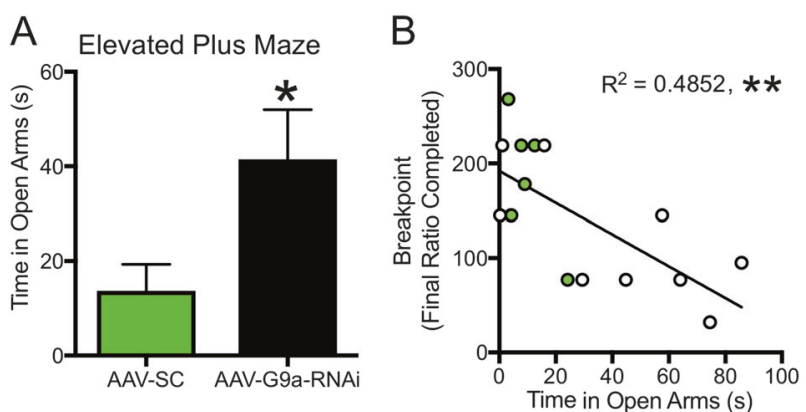

Fig. 4 AAV-RNAi-mediated G9a knockdown in NAcSh leads to lasting decreases in anxiety-like behaviors in cocaine-experienced rats. a Time spent in the open arms of the elevated plus maze. (AAVSC: $n=9$, AAV-G9a-RNAi: $n=9$ ) Data are expressed as mean $+/-$ s.e.m. ${ }^{*} p<0.05$, and ${ }^{* *} p<0.01$ compared with AAV-SC controls. b Correlation between time spent in the open arms and breakpoints on the progressive ratio test at $0.75 \mathrm{mg} / \mathrm{kg} / \mathrm{inf}$. AAV-SC controls are represented by green circles-note that 2 AAV-SC rats overlap almost completely-and AAV-G9a-RNAi are represented by white circles. ${ }^{*} p<0.05$ represents a significant linear regression

post-expression effects are limited to footshock stress-induced reinstatement and elevated anxiety scores [7]. In contrast, continuous AAV-RNAi-mediated G9a knockdown throughout extinction/reinstatement/anxiety testing reduces all of these behaviors, probably due to the persistent reduction of G9a levels throughout the cocaine abstinence, extinction, and testing periods. It could be interesting to determine whether decreasing G9a levels after stable cocaine self-administration produces similar effects on cocaine-seeking behavior, or whether an interaction between reduced G9a levels and cocaine exposure during selfadministration is required. Given that normal functioning of the NAcSh is necessary for dopamine-related memory consolidation [26-29], the decrease in initial extinction produced by NAcSh G9a knockdown may reflect impairment in the formation of contextual memories during prior cocaine self-administration, rather than a direct effect on extinction or reinstatement responding. In any event, the fact that reduced G9a activity counters the ability of multiple conditioned environmental, pharmacological, and stressful stimuli to trigger cocaine seeking suggests that blocking G9a activity may have widespread utility in reducing relapse vulnerability in individuals suffering from cocaine use disorder.

It is intriguing that G9a levels in the NAcSh exert such strong control over anxiety-like behavior in both cocaine-experienced and drug-naïve rats. AAV-RNAi-mediated G9a knockdown decreases the latency to enter the open arms - indicative of a powerful anxiolytic effect [30] - and entirely consistent with a reduction in reinstatement of cocaine-seeking by intermittent footshock stress. In addition, marble burying is decreased by G9a knockdown, another measure of anxiety-like behavior that is reversible by anxiolytics [31, 32]. G9a in the NAcSh thus likely exerts a bi-directional influence on anxiety as our previous study showed enduring anxiogenic effects with G9a overexpression [7]. This also suggests that the alterations in anxiety could be causing the changes in cocaine-taking and cocaine-seeking behaviors. Clinical studies find evidence for high co-morbidity for addiction and anxiety [33-35], but causal links are difficult to establish. In rodent studies, however, there is evidence for functional interactions in both directions. For instance, high anxiety phenotypes predict increased cocaine self-administration in rats [36], and anxiolytic drugs can reduce cocaine self-administration [37]. Conversely, chronic cocaine exposure itself also increases anxiety phenotypes [38-42]. In any event, the ability of reduced G9a activity to attenuate both cocaine seeking and anxiety-like behavior suggests that reductions in G9a in NAcSh could be therapeutic for co-morbid cocaine addiction and anxiety. 
A

\begin{tabular}{|c|c|c|c|c|c|}
\hline Stage & Sur Rec & EPM & Marble burying & Locomotor & Tissue Collection \\
\hline Time & $1 d 3 w k$ & $1 d$ & $1 d$ & $1 d$ & $1 d$ \\
\hline
\end{tabular}

Viral Expression $\|= \pm$ -

B

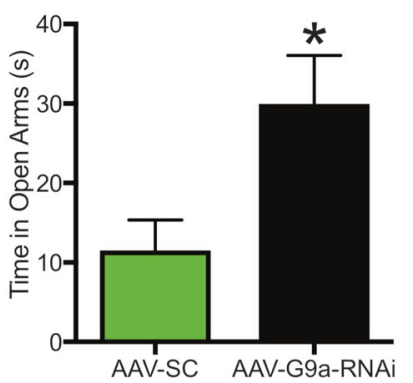

E



C

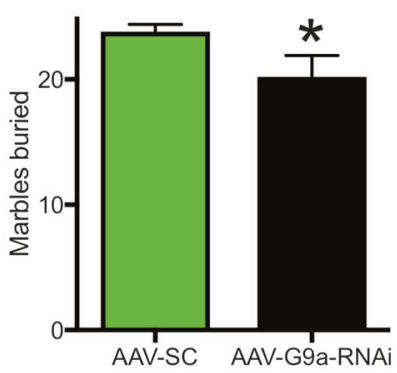

F

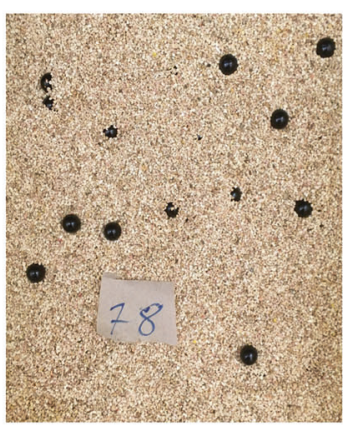

D



G
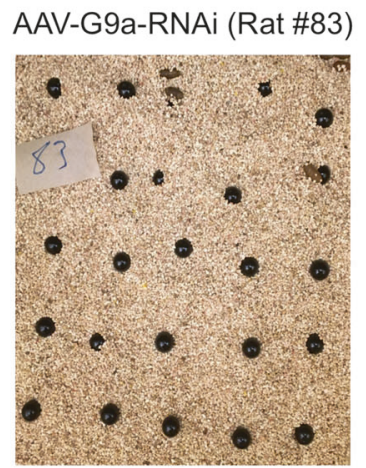

Fig. 5 AAV-RNAi-mediated G9a knockdown in NAcSh leads to decreases in basal anxiety-like behaviors in drug-naïve rats. a Experimental timeline. b Time spent in the open arms of the elevated plus maze. c Number of marbles buried after 15 min (AAV-SC: $n=5$, AAV-G9a-RNAi: $n=5)$. d Correlation between time spent in the open arms and number of marbles buried. AAV-SC controls are represented by green circles and AAV-G9a-RNAi are represented by white circles (e) Representative images of marbles before testing began compared to an (f) AAV-SC control and a (g) AAV-G9a-RNAi rat. For $\mathbf{b}$ and $\mathbf{c}$, data are expressed as mean $+/-$ s.e.m. ${ }^{*} p<0.05$ compared with AAV-SC controls. For $\mathbf{d}$, ${ }^{*} p<0.05$ represents a significant linear regression

These findings also suggest that the down-regulation of G9a in the NAc produced by repeated cocaine use in both selfadministrating rodents [11] and humans [10] is counter-adaptive to the formation of comorbid addiction-anxiety states, as we show here that further decreases in G9a in this region reduce selfadministration and anxiety behaviors. It is interesting that Maze et al. [11]. found that localized knockout of G9a in NAc neurons increased cocaine-conditioned place preference in mice, whereas G9a overexpression diminished cocaine place preference, diametrically opposite to the effects of NAc G9a on cocaine reinforcement in self-administering rats reported here. One major difference in these studies is the level of experience with cocaine since the mice were cocaine-naïve before place preference testing, whereas rats in our study had several weeks of cocaine self-administration before the testing phase began. However, we previously found that G9a overexpression produces the same increases in cocaine-sensitivity, motivation, and stress-induced reinstatement regardless of whether it was overexpressed during or after initial acqusition and stabilization of cocaine self-administration behavior [7]. These data suggest that the level of cocaine experience does not likely explain the differences between these studies $[7,11]$. Another possibility is that NAc G9a could differentially alter behavior in rats and mice as behavioral differences between cocaine place preference and cocaine self-administraion have been observed, even within the same species $[23,43]$. Thus, for example, while reductions in G9a levels could promote the positive rewarding influence of passive cocaine administration in conditioned place preference by reducing the anxiogenic component of initial cocaine exposure $[40,41]$, these same anxiolytic effects may reduce motivation for cocaine through attenuation of negative reinforcement mechanisms that otherwise are alleviated through voluntary self-administration behavior.

To conclude, these data show that an RNAi-mediated knockdown of G9a in NAcSh exerts powerful reductions in cocaine selfadministration, cocaine seeking, and basal anxiety-related behavioral responses. Given that chronic cocaine [11] and morphine [12] both reduce $\mathrm{G} 9 \mathrm{a}$ and/or $\mathrm{H} 3 \mathrm{~K} 9 \mathrm{me} 2$ in NAc, these G9a reductions may represent a common adaptive response of NAc neurons to compensate for the negative effects of chronic drug use on the brain. In this sense, there would seem to be numerous-yet mostly unidentified-genes normally suppressed by G9a that act to counter the concomitant increase in addiction-promoting gene expression that also are unmasked by global G9a reductions, e.g., increases in CREB-regulated and $\triangle$ FosB-regulated genes. Future studies could utilize this AAV-G9a-RNAi vector to identify promoters with reduced $\mathrm{H} 3 \mathrm{~K} 9 \mathrm{me2}$, and also the striatal cell types where G9a reductions exert this counter-addictive property. For example, previous work has shown differential effects of developmental deletion of G9a in D1 or D2 striatal neurons in cocaine-conditioned place preference [10]. This study found that G9a deletion in D1 neurons reduces cocaine place preference, and so unrepressed genes in these neurons may also reduce cocaine self-administration and relapse behaviors. Moreover, these D1 neurons may also mediate anxiolytic effects, since intra-NAcSh administration of a D1 (but not D2) antagonist produces anti-anxiety-like actions [44]. 
Conversely, repression of gene expression via overexpression of the class III histone deacetylase SIRT in D1 but not D2 cells increases anxiety-like behavior [45]. Together, these findings support a common cellular mechanism for the functional interplay between reduced addictive and anxiety behaviors. They could also pave the way for clinical treatments that treat both addiction and anxiety disorders in comorbid patients.

\section{FUNDING AND DISCLOSURE}

This work was funded by T32 DA 007290, P01 DA 008227, and R01 DA032708. The authors declare no competing interests.

\section{ACKNOWLEDGEMENTS}

We would like to thank Shari Birnbaum, Ph.D. at the University of Texas Southwestern for her assistance with the elevated plus maze assay. We would also like to thank Dr. Carmela Reichel, Dr. Jacqueline McGinty, Dr. Rhett Reichard, Angela Kearns, and Jordan Hopkins at the Medical University of South Carolina for their assistance with the basal anxiety experiments.

\section{ADDITIONAL INFORMATION}

Publisher's note: Springer Nature remains neutral with regard to jurisdictional claims in published maps and institutional affiliations.

\section{REFERENCES}

1. Back SE, Brady KT. Anxiety disorders with comorbid substance use disorders: diagnostic and treatment considerations. Psychiatr Ann. 2008;38:724-9.

2. Vorspan F, Mehtelli W, Dupuy G, Bloch V, Lepine JP. Anxiety and substance use disorders: co-occurrence and clinical issues. Curr Psychiatry Rep. 2015;17:4.

3. Dennis ML, Foss MA, Scott CK. An eight-year perspective on the relationship between the duration of abstinence and other aspects of recovery. Eval Rev. 2007;31:585-612.

4. Johnson BA, et al. Topiramate for the treatment of cocaine addiction: a randomized clinical trial. JAMA Psychiatry. 2013;70:1338-46.

5. Martell BA, Mitchell E, Poling J, Gonsai K, Kosten TR. Vaccine pharmacotherapy for the treatment of cocaine dependence. Biol Psychiatry. 2005;58:158-64.

6. Covington $\mathrm{HE} 3 \mathrm{rd}$, et al. A role for repressive histone methylation in cocaineinduced vulnerability to stress. Neuron. 2011;71:656-70.

7. Anderson EM, et al. Overexpression of the histone dimethyltransferase G9a in nucleus accumbens shell increases cocaine self-administration, stress-induced reinstatement, and anxiety. J Neurosci. 2018;38:803-13.

8. Robison AJ, Nestler EJ. Transcriptional and epigenetic mechanisms of addiction. Nat Rev Neurosci. 2011;12:623-37.

9. Anderson, EM et al. It's a complex issue: emerging connections between epigenetic regulators in drug addiction. Eur J Neurosci. (2018). Epub ahead of print.

10. Maze I, et al. G9a influences neuronal subtype specification in striatum. Nat Neurosci. 2014;17:533-9.

11. Maze I, et al. Essential role of the histone methyltransferase G9a in cocaineinduced plasticity. Science. 2010;327:213-6.

12. Sun $\mathrm{H}$, et al. Morphine epigenomically regulates behavior through alterations in histone $\mathrm{H} 3$ lysine 9 dimethylation in the nucleus accumbens. J Neurosci. 2012;32:17454-64.

13. Qiang M, Denny A, Lieu M, Carreon S, Li J. Histone H3K9 modifications are a local chromatin event involved in ethanol-induced neuroadaptation of the NR2B gene. Epigenetics. 2011;6:1095-104.

14. Zhang Z, et al. MeCP2 repression of G9a in regulation of pain and morphine reward. J Neurosci. 2014;34:9076-87.

15. Anderson, EM et al. BDNF-TrkB controls cocaine-induced dendritic spines in rodent nucleus accumbens dissociated from increases in addictive behaviors. Proc Natl Acad Sci USA. 2017;114:9469-74.

16. Graham DL, et al. Dynamic BDNF activity in nucleus accumbens with cocaine use increases self-administration and relapse. Nat Neurosci. 2007;10:1029-37.

17. Graham DL, et al. Tropomyosin-related kinase B in the mesolimbic dopamine system: region-specific effects on cocaine reward. Biol Psychiatry. 2009;65:696-701.

18. Schaefer A, et al. Control of cognition and adaptive behavior by the GLP/G9a epigenetic suppressor complex. Neuron. 2009;64:678-91.
19. Wang, DY, et al. Inhibition of the G9a/GLP histone methyltransferase complex modulates anxiety-related behavior in mice. Acta Pharmacol Sin. https://doi.org/ 10.1038/aps.2017.190 (2018).

20. Edwards S, Bachtell RK, Guzman D, Whisler KN, Self DW. Emergence of contextassociated GluR(1) and ERK phosphorylation in the nucleus accumbens core during withdrawal from cocaine self-administration. Addict Biol. 2011;16:450-7.

21. Edwards S, Graham DL, Bachtell RK, Self DW. Region-specific tolerance to cocaineregulated CAMP-dependent protein phosphorylation following chronic selfadministration. Eur J Neurosci. 2007;25:2201-13.

22. Edwards S, Whisler KN, Fuller DC, Orsulak PJ, Self DW. Addiction-related alterations in D1 and D2 dopamine receptor behavioral responses following chronic cocaine self-administration. Neuropsychopharmacology. 2007;32:354-66.

23. Larson EB, et al. Overexpression of CREB in the nucleus accumbens shell increases cocaine reinforcement in self-administering rats. J Neurosci. 2011;31:16447-57.

24. Penrod RD, Wells AM, Carlezon WA Jr., Cowan CW. Use of adeno-associated and herpes simplex viral vectors for in vivo neuronal expression in mice. Curr Protoc Neurosci. 2015;73:31-31. 437

25. Paxinos, G and Watson, C. The rat brain in stereotaxic coordinates. Academic Press; Cambridge, Massachusetts 1997.

26. LaLumiere RT, Nawar EM, McGaugh JL. Modulation of memory consolidation by the basolateral amygdala or nucleus accumbens shell requires concurrent dopamine receptor activation in both brain regions. Learn Mem. 2005; 12:296-301.

27. Hafenbreidel M, Rafa Todd C, Mueller D. Infralimbic GluN2A-Containing NMDA receptors modulate reconsolidation of cocaine self-administration memory. Neuropsychopharmacology. 2017:42:1113-25.

28. Li G, et al. Inhibition of actin polymerization in the NAc shell inhibits morphineinduced CPP by disrupting its reconsolidation. Sci Rep. 2015;5:16283.

29. Rasekhi K, Oryan S, Nasehi M, Zarrindast MR. Involvement of the nucleus accumbens shell glutamatergic system in ACPA-induced impairment of inhibitory avoidance memory consolidation. Behav Brain Res. 2014;269:28-36.

30. Handley SL, Mithani S. Effects of alpha-adrenoceptor agonists and antagonists in a maze-exploration model of 'fear'-motivated behaviour. Naunyn Schmiede Arch Pharmacol. 1984;327:1-5.

31. Archer T, Fredriksson A, Lewander T, Soderberg U. Marble burying and spontaneous motor activity in mice: interactions over days and the effect of diazepam. Scand J Psychol. 1987;28:242-9.

32. Broekkamp CL, Rijk HW, Joly-Gelouin D, Lloyd KL. Major tranquillizers can be distinguished from minor tranquillizers on the basis of effects on marble burying and swim-induced grooming in mice. Eur J Pharmacol. 1986;126:223-9.

33. Jakuszkowiak-Wojten K, Landowski J, Wiglusz MS, Cubala WJ. Impulsivity in anxiety disorders. A critical review. Psychiatr Danub. 2015;27(Suppl 1):S452-455.

34. Ersche KD, et al. Cognitive dysfunction and anxious-impulsive personality traits are endophenotypes for drug dependence. Am J Psychiatry. 2012;169: 926-36.

35. Voon V, Dalley JW. Translatable and back-translatable measurement of impulsivity and compulsivity: convergent and divergent processes. Curr Top Behav Neurosci. 2016;28:53-91.

36. Dilleen $\mathrm{R}$, et al. High anxiety is a predisposing endophenotype for loss of control over cocaine, but not heroin, self-administration in rats. Psychopharmacology. 2012:222:89-97.

37. Augier E, Vouillac C, Ahmed SH. Diazepam promotes choice of abstinence in cocaine self-administering rats. Addict Biol. 2012;17:378-91.

38. Morton WA. Cocaine and psychiatric symptoms. Prim Care Companion J Clin Psychiatry. 1999;1:109-13.

39. Wenzel JM, et al. Noradrenergic beta-receptor antagonism within the central nucleus of the amygdala or bed nucleus of the stria terminalis attenuates the negative/anxiogenic effects of cocaine. J Neurosci. 2014;34:3467-74.

40. Ettenberg A. Opponent process properties of self-administered cocaine. Neurosci Biobehav Rev. 2004:27:721-8.

41. Guzman D, Ettenberg A. Runway self-administration of intracerebroventricular cocaine: evidence of mixed positive and negative drug actions. Behav Pharmacol. 2007; $18: 53-60$.

42. Guzman D, Ettenberg A. Heroin attenuates the negative consequences of cocaine in a runway model of self-administration. Pharmacol Biochem Behav. 2004:79:317-24.

43. Carlezon WA Jr., et al. Regulation of cocaine reward by CREB. Science. 1998;282:2272-5.

44. Zarrindast $M R$, et al. Involvement of rat dopaminergic system of nucleus accumbens in nicotine-induced anxiogenic-like behaviors. Brain Res. 2012;1460:25-32.

45. Kim HD, et al. SIRT1 mediates depression-like behaviors in the nucleus accumbens. J Neurosci. 2016:36:8441-52. 